\title{
Downregulation of RLIP76 is associated with vincristine resistance in human colorectal cancer HCT-8/VCR cells
}

\author{
WENWEN WANG ${ }^{1}$, JUAN LIU $^{1}$, JIANNI QI ${ }^{2}$, JUNYONG ZHANG $^{1}$, \\ QIANG ZHU ${ }^{1}$, JINCAI MA ${ }^{3}$ and CHENGYONG QIN ${ }^{1}$ \\ ${ }^{1}$ Department of Gastroenterology, ${ }^{2}$ Central Laboratory, Shandong Provincial Hospital \\ Affiliated to Shandong University, Jinan, Shandong 250021; ${ }^{3}$ Department of Gastroenterology, \\ The Fifth People's Hospital of Jinan, Jinan, Shandong, 250021, P.R. China
}

Received April 14, 2016; Accepted July 27, 2016

DOI: $10.3892 /$ ijo.2016.3672

\begin{abstract}
RLIP76 is an anti-apoptotic transporter, participating in the multi-specific drug transport and resistance. In the absence of chemotherapy drugs, the knockout or inhibition of RLIP76 leads to pronounced tumor regression. RLIP76 transports anthracycline and vinca alkaloid drugs and mediates the resistance to these drugs. However, functions of RLIP76 in drug resistance colorectal cancer remain unclear. HCT-8 and the vincristine (VCR)-resistant colorectal cancer cell line HCT-8/VCR (HCT-8/V) were used in the present study. The effects of RLIP76 knockdown by the lentivirus were examined in cultured cells, including growth, apoptosis, invasion, and signaling pathways by qRT-PCR, western blot analysis and Transwell assay. The relative level of RLIP76 in HCT-8 and HCT-8/V was assessed by western blot analysis, finding RLIP76 was overexpressed in HCT-8/V. Then, HCT-8/V cancer cells were transfected with lentivirus encoding RLIP76-specific shRNA (KD) and the control (NC), and no significant difference of RLIP76 level between the NC cells and cells without transfection was found, but the relative mRNA level decreased to $0.277 \pm 0.016$ and protein level also reduced in KD cells. Cell functions changed after RLIP76 knockdown in HCT-8/V. The $\mathrm{IC}_{50}$ of VCR decreased from $164.4 \pm 1.734$ to $13.95 \pm 2.008(\mu \mathrm{g} / \mathrm{ml})(\mathrm{P}<0.05)$ in cell culture. The cell number reduced from $329.67 \pm 20.23$ to $176.33 \pm 2.52$ $(\mathrm{P}<0.05)$ in migration assay and from $294.67 \pm 30.07$ to $153 \pm 22.11(\mathrm{P}<0.05)$ in invasion assay. Moreover, apoptotic proteins, including cleaved-caspase-8, cleaved-caspase-9, cleaved-Parp and Bax increased. The phosphorylation level of Erk also reduced significantly. The present study showed that RLIP76 is a key effector of cancer cell survival, invasion, and
\end{abstract}

Correspondence to: Dr Chengyong Qin, Department of Gastroenterology, Shandong Provincial Hospital Affiliated to Shandong University, 324 Jingwu Weiqi Road, Jinan, Shandong 250021, P.R. China

E-mail: qinchengyongdaoshi@163.com

Key words: colorectal cancer, RLIP76, MDR, signaling pathways, apoptosis, vincristine migration and possibly an important target to improve drug resistance and tumor treatment.

\section{Introduction}

Colorectal cancer is the leading, and second leading cause of death in developed and developing countries, respectively. The survival rate of colorectal cancer patients has increased due to the early diagnosis and treatment strategies (1). However, the 5-year survival rate still remains at less than $60 \%$ (2). For colorectal cancer, surgery is the primary treatment method. While during the later phases, for example, the node-positive stage III, adjuvant chemotherapy is necessary (1). Vincristine (VCR) is widely used in tumor treatment. However, in colorectal cancer chemotherapy treatment, the development of acquired multidrug-resistance (MDR) to conventional chemotherapeutics has been the main restriction $(3,4)$. MDR is associated with decrease of drug accumulation in cell due to active energy-dependent efflux of drugs or metabolites (5-8). Given this premise, novel treatment strategies which could help overcome MDR, as well as increase tumor cell response to chemotherapy drugs are greatly needed.

RLIP76 is a 76-kDa splice variant, which was encoded by the human gene RALBPI (18p11.22). It is a Ral-interacting protein of $76 \mathrm{kDa}$, also known as RalBP1. It was identified as a Ral GTPase effector protein that connects the Ral with Rho pathways originally (9-11). This protein participates in the ATP hydrolysis-dependent movement of substances, including glutathione conjugates (GS-E) and chemotherapy drugs, out of cells (12-14). GS-Es are toxic to the cells and need to be transported out of cells in order to keep cells from death. As a result, RLIP76 mediates resistance to its substrates, which range from weakly cationic compounds, such as doxorubicin (DOX), vinblastine (VBL), vincristine (VCR), vinorelbine (VRL) (15-17), colchicine, sunitinib and sorafenib $(11,18)$, to anionic metabolites, including glutathione conjugates of electrophiles (19). Knockout of RLIP76 with targeting antibodies or antisense molecules can be able to increase the sensitivity to radiation and chemotherapy of tumors and cause solid tumors regression in non-small cell lung cancer, colorectal carcinomas (20), prostate cancer (21), and B16 melanomas (22) in 
mice and pancreatic cancer (23), glioblastoma (24) in human. One of the mechanisms is that knockout of RLIP76 increased cellular accumulation of chemotherapy drugs.

Most early studies concentrated on research of the transport functions of RLIP76, whereas growing evidence has shown that RLIP76 is necessary in a variety of cellular functions, such as mitosis, proliferation, differentiation, apoptosis and endocytosis (25-27). It takes part in the formation of multi-functional protein complexes, like the mitotic spindle and the receptor signaling complexes of EGF, TGF- $\beta$, insulin and clathrin-dependent endocytosis (28-30) and determine the rate of receptor-ligand signaling. RLIP76 exists in many human tissues, including liver, heart and ovary, but overexpressed in various types of cancer cells, including lung and ovarian carcinomas and melanomas $(16,31,32)$. Blocking RLIP76 with targeting antibodies or knockout RLIP76 with antisense results in apoptosis in many types of cancer cells in vitro $(11,18,33-37)$, and sensitivity to apoptosis on RLIP76 depletion in malignant cells is greater than in non-malignant cells (22). RLIP76 belongs to Ras family and transmits signals from Ral to the downstream protein, cdc42. Activation of the Rho family G-protein cdc42, has been shown to induce apoptosis (38). RLIP76 is also involved in various cellular signaling pathways, such as PI3K/Akt and Erk signaling pathway which regulates resistance to chemo-radiotherapy and basal survival in a variety of cancers (25-27). Phosphorylation of Erk and PI3K is markedly and consistently decreased in all human kidney cancer cell lines due to RLIP76 deletion (13). These data show that RLIP76 is a potential target for tumor treatment, but the roles of RLIP76 in colorectal cancer, especially in multidrug resistance (MDR) of colorectal cancer are still unknown.

In the present study, we verified RLIP76 level in MDR cancer cells and cancer cells without drug resistance. Then, the function of RLIP76 on chemotherapy, migration, invasion, apoptosis and signaling pathway was detected after knockdown of RLIP76. Our findings provide important insights into VCR resistance and highlight RLIP76 as a novel molecular target that can be specifically inhibited to sensitize colorectal cancer cells to VCR.

\section{Materials and methods}

Cell culture and reagents. The human colorectal cancer HCT- 8 cell line and the MDR HCT-8/V cell line were obtained from Nanjing KeyGen Biotech. Co., Ltd (Nanjing, China). Cells were grown in RPMI-1640 medium supplemented with $10 \%$ heat-inactivated fetal bovine serum (FBS), $2 \mathrm{mM}$ glutamine, $100 \mu / \mathrm{ml}$ penicillin, and $100 \mathrm{ng} / \mathrm{ml}$ streptomycin (Invitrogen, Carlsbad, CA, USA) at $37^{\circ} \mathrm{C}$ in a $5 \% \mathrm{CO}_{2}$ humidified atmosphere. HCT-8/V cells were routinely maintained in a medium containing $1000 \mathrm{ng} / \mathrm{l} \mathrm{VCR}$ (vincristine sulfate; Dalian Meilun Biotech Co., Ltd., Dalian, China) and incubated in a drug-free medium for at least a week before use.

Drug sensitivity was determined by Cell Counting kit-8 (CCK-8). Cells were counted and plated into 96-well plates at a density of $3 \times 10^{3}(1.2 \mu \mathrm{l})$ cells/well. The VCR used was dissolved with RPMI-1640 medium and then diluted at different concentrations including 5, 10, 50, 100, 200, 500 and $1000 \mu \mathrm{g} / \mathrm{ml}$ with RPMI-1640 medium supplemented with 10\% FBS, 2 mM gluta- mine, $100 \mu / \mathrm{ml}$ penicillin and $100 \mathrm{ng} / \mathrm{ml}$ streptomycin. Cells were cultured overnight and then were cultured in the medium at various concentrations of VCR for $48 \mathrm{~h}$, then $20 \mu \mathrm{l}$ of CCK-8 (Beyotime Institute of Biotechnology, Haimen, China) was added to each well, following incubation for $4 \mathrm{~h}$ at $37^{\circ} \mathrm{C}$. Each solution was subjected to spectrophotometry at $450 \mathrm{~nm}$ in a Multiskan Ascent microplate reader (Thermo Fisher Scientific, Vantaa, Finland). The drug sensitivity is expressed as the half maximal inhibitory concentration $\left(\mathrm{IC}_{50}\right)$ for each of the cell lines, which represents the concentration of the drug that caused a $50 \%$ reduction in the absorbance at $450 \mathrm{~nm}$ relative to the untreated cells (control). GraphPad Prism 5 was used to calculate the $\mathrm{IC}_{50}$. In cell proliferation assay, $5 \times 10^{3}$ cells were plated into 96-well plates. Each well contained medium supplemented with $10 \%$ FBS. The cultures were stained using a Cell Counting kit- 8 at various time-points.

Transwell assay. Cell migration and invasion were detected by a Transwell assay. Cells were starved overnight in serum-free medium, trypsinized, and washed three times in RPMI-1640 medium without FBS. For migration, $8.76 \mu 1$ cells $\left(1 \times 10^{5}\right)$ were seeded into the upper chambers in $200 \mu \mathrm{l}$ serum-free media without Matrigel membrane. In addition, the lower chambers were loaded with $600 \mu 1$ RPMI-1640 supplemented with $10 \%$ FBS. After $24 \mathrm{~h}$, the cells in the upper chambers that had not migrated were removed with a cotton swab. For the invasion assay, colorectal cancer cells $\left(2 \times 10^{5}\right)$ were seeded into the upper chambers with a Matrigel $(8-\mu \mathrm{m}$ pore size; BD Biosciences, San Jose, CA, USA) membrane and after $48 \mathrm{~h}$, the cells in the upper chambers that had not migrated were removed with a cotton swab. The cells on the lower surface of the membrane were fixed in formaldehyde and stained with hematoxylin staining solution. Then, the cells were counted and photographed.

Western blot analysis. The total protein was extracted from the cancer cells, and the proteins were separated by sodium dodecyl sulfate-polyacrylamide gel electrophoresis (SDS-PAGE). Crude fraction containing $40 \mathrm{mg}$ of proteins were subjected to SDS-PAGE and proteins were transferred onto PVDF membrane. The detection of $\beta$-actin $(1: 10,000$; Santa Cruz Biotechnology, Santa Cruz, CA, USA) on the same membrane was used as the internal control. Specific antibodies for RLIP76 (ab133549; monoclonal, 1/10,0001/50,000; Abcam, Cambridge, MA, USA), caspase-3 (\#9662; 1:1,000, polyclonal; Cell Signaling Technology, Danvers, MA, USA), caspase-8 (\#9746; 1:1,000; monoclonal; Cell Signaling Technology), caspase-9 (\#9508; 1:1,000; monoclonal; Cell Signaling Technology), PARP (\#9542; 1:1,000; monoclonal; Cell Signaling Technology), phosphorylated Erk (\#4370; 1:2,000, monoclonal; Cell Signaling Technology) and Erk (\#4695; 1:1,000, monoclonal; Cell Signaling Technology) were used for the immunodetection of the corresponding proteins. Subsequently, HRP-conjugated secondary antibodies (1:10,000; Beijing Zhongshan Golden Bridge Biotechnology Co., Ltd., Beijing, China), followed by enhanced chemiluminescence (Millipore Corp., Billerica, MA, USA), were used. The same amount of protein was used each time.

Lentiviral infection and stable cell line selection. The lentivirus that encoded RLIP76-specific shRNA and the scrambled 

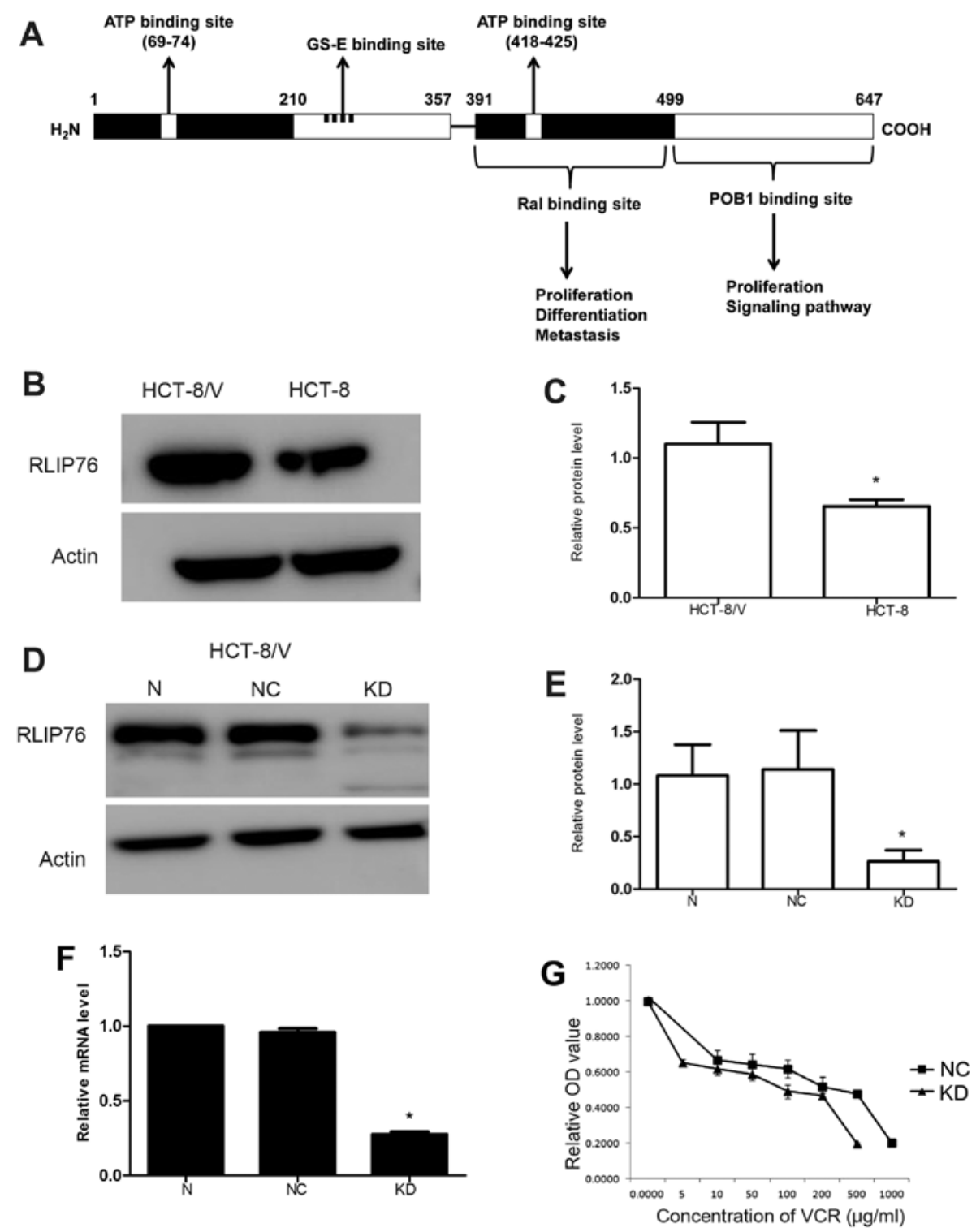

Figure 1. Knockdown of RLIP76 and $\mathrm{IC}_{50}$ of VCR. (A) Schematic representation of RLIP76. There are different domains of RLIP76 including ATP binding domain, GS-E binding site, Ral binding domain and POB1 binding domain (48). (B and C) Comparison of RLIP76 levels in HCT-8 and HCT-8/V by western blot analysis. Higher RLIP76 expression occurred in HCT-8/V. (D and E) Western blot analysis demonstrated that RLIP76 decreased in HCT-8/V after transfection ( $\mathrm{P}<0.05)$ on protein level. (F) Quantitative RT-PCR demonstrated that RLIP76 decreased in HCT-8/V after transfection *P<0.05) on RNA level. Data are means \pm standard deviations of values from triplicate experiments. (G) Cells were cultured with different concentration of VCR. The absorbance value was measured after $48 \mathrm{~h}$. Results of CCK-8 demonstrated the $\mathrm{IC}_{50}$ of HCT-8/V to VCR after transfection ( $\left.{ }^{*} \mathrm{P}<0.05\right)$.

shRNA lentivirus were generated by GenomeDitech Co., Ltd., Shanghai, China. MDR colorectal cancer cells (HCT-8/V) were infected with recombinant shRNA that was specific for RLIP76 lentiviral stocks or scrambled shRNA lentiviral stocks. qRT-PCR and western blot analysis were used to select stable RLIP76 knockdown cell lines (KD) and the control cell lines (NC). The lentiviral vectors expressed the green fluorescent protein, which allowed for the measurement of infection efficiency in the transfected cells.

Quantitative RT-PCR ( $q R T-P C R)$ analysis. The total cellular RNA was extracted with the TRIzol (Takara Bio, Dalian, China) reagent and reverse transcribed to cDNA according to the manufacturer's protocols. The qPCR products were detected with SYBR-Green (Takara) in a LightCycler ${ }^{\circledR} 480$ Real-Time PCR System (Roche Diagnostics). The $\beta$-actin gene was amplified as an internal control. The primers for RLIP76: 5'-ggCATgAAgTgTgAAggCATCTAC-3' and 5'-CT CgCAAATACTgCTTCAgCAAAC-3' were used for qPCR.

Statistical analysis. The data were evaluated with a two-tailed unpaired Student's t-test or with a two-tailed paired Student's t-test. Values with $\mathrm{P}<0.05$ were considered to be statistically significant.

\section{Results}

RLIP76 is overexpressed in HCT-8/V. RLIP76 contains ATP binding domain, GS-E binding site, Ral binding domain and POB1 binding domain, which indicates it may play a role in proliferation, metastasis, ATP-dependent transport and apoptosis (Fig. 1A). Vincristine (VCR) is one of the substrates of 

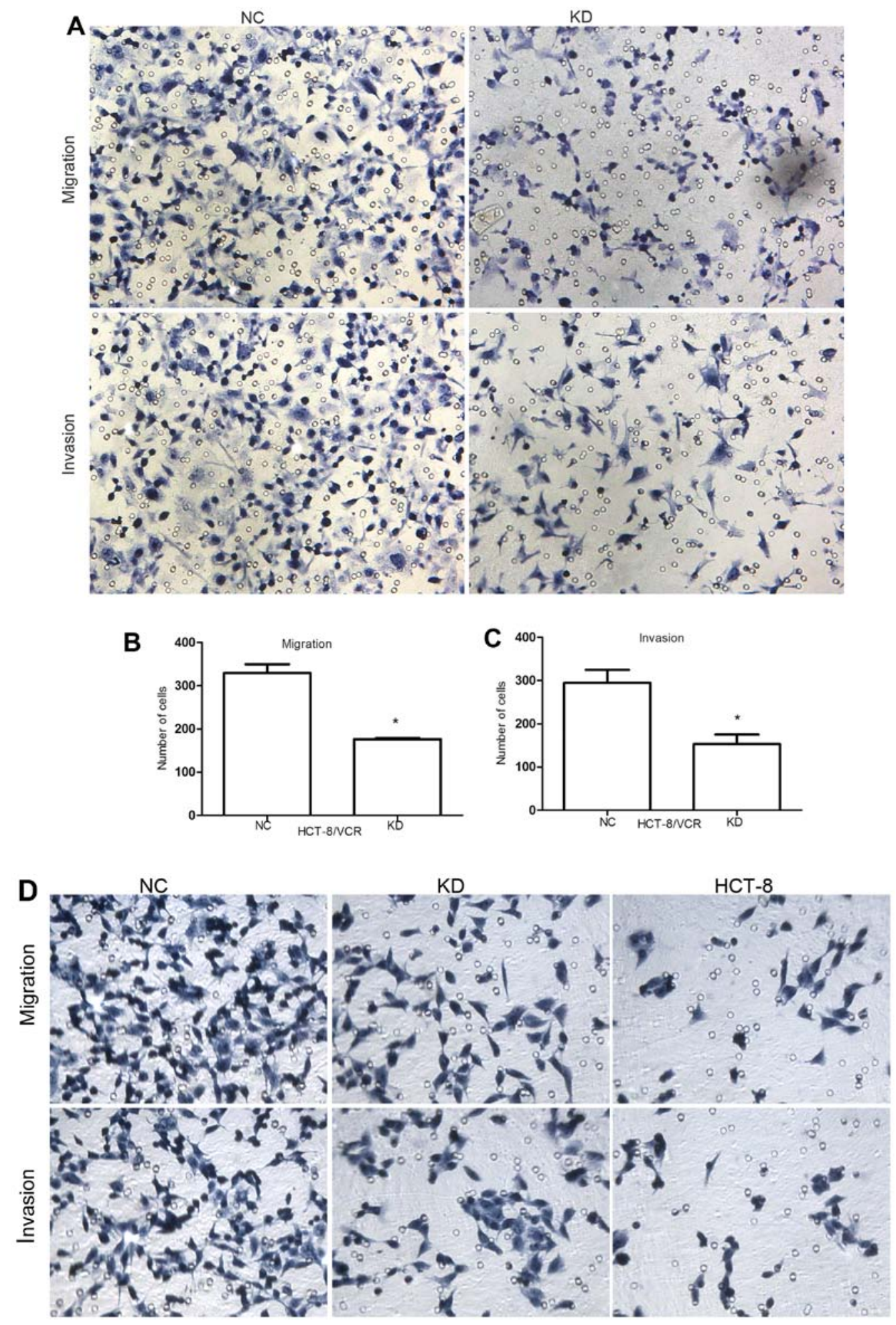

Figure 2. (A-C) Migration and invasion. The number of cells that migrated across the membrane without or with the cold Matrigel coating. Cell migration decreased from $329.67 \pm 20.23$ to $176.33 \pm 2.52\left({ }^{*} \mathrm{P}<0.05\right.$ ) and cell invasion reduced from $294.67 \pm 30.07$ to $153 \pm 22.11$ ("P $<0.05$ ). (D) Migration and invasion assay were performed to compare with HCT-8. The migration and invasion levels did not decrease similarly to HCT-8 after the RLIP76 knockdown.

RLIP76. The level of RLIP76 was detected by western blot analysis, and the HCT-8/V cell line exhibited higher expression compared with that of the HCT-8 cell line (Fig. 1B andC; $\mathrm{P}<0.05)$.

RLIP76-specific shRNA decreases RLIP76 expression in $H C T-8 / V$. Lentiviral vector-mediated RNA interference tech- nology was used to infect the HCT-8/V cells with the negative control (NC) and RLIP76-specific shRNA lentivirus (KD) to generate stable cell lines. Transfection of HCT-8/V cells with RLIP76-specific shRNA lentivirus (KD) markedly downregulated the RLIP76 protein levels compared with NC cells to $0.264 \pm 0.106$ as determined by western blot analysis (Fig. 1D and $\mathrm{E} ; \mathrm{P}<0.05$ ). No significant difference of relative RLIP76 


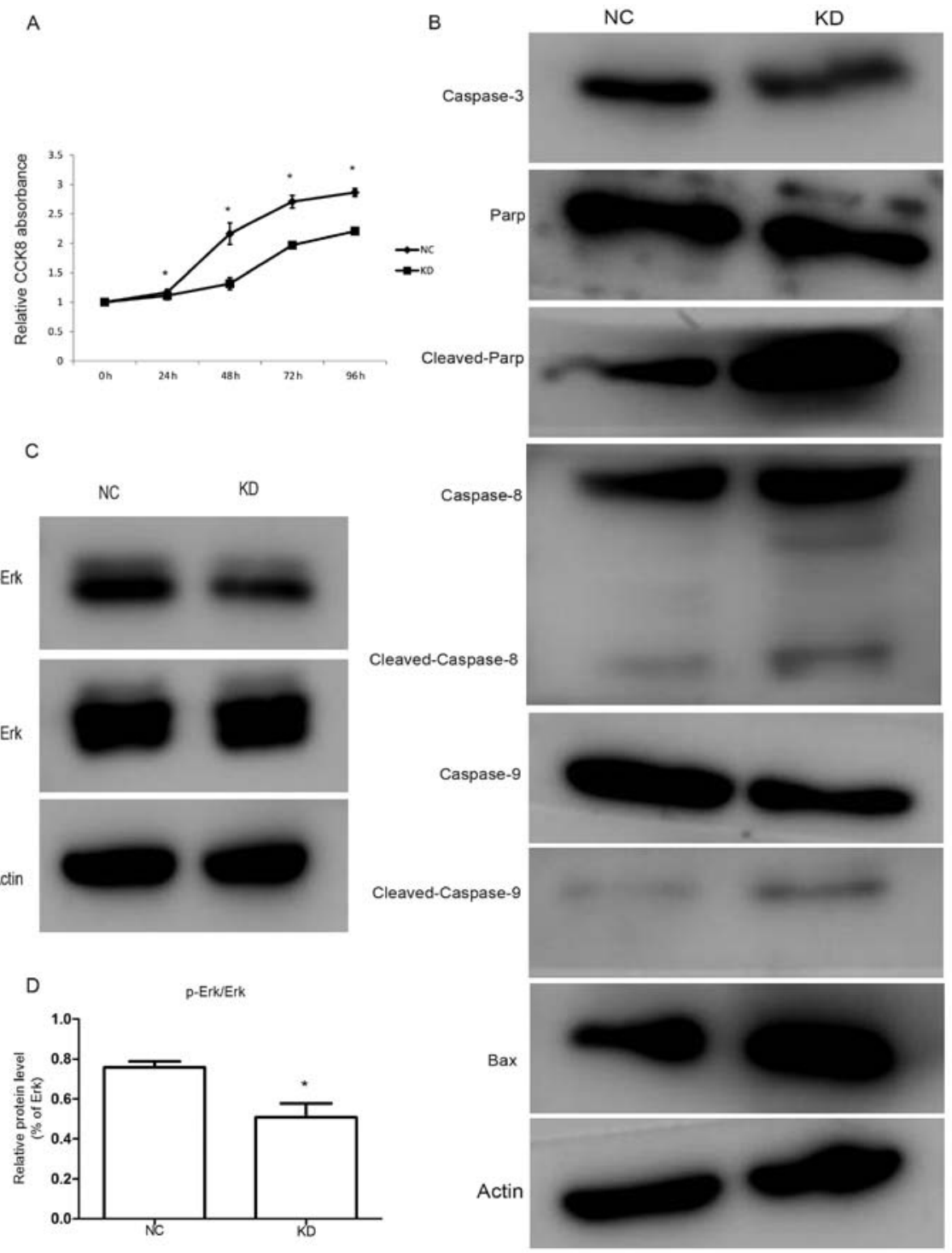

Figure 3. RLIP76 regulated proliferation and apoptosis through Erk pathway. (A) Cells were plated into 96-well plates. The cultures were stained using a Cell Counting kit-8 at various time-points. The KD cells showed obvious decrease of growth compared with the NC cells, especially at the $48 \mathrm{~h}$ time-point $($ ( $\mathrm{P}<0.05)$. (B) The level of caspase-3 in KD cells was markedly decreased compared with the controls, whereas the levels of Bax, cleaved-PARP, cleavedcaspase-8 and cleaved-caspase-9 increased in western blot analysis. (C and D) In KD cells, the level of phosphorylated Erk decreased ("P<0.05) whereas the unphosphorylated Erk remained constant.

mRNA level was found by qRT-PCR between cells without transfection and the NC cells. However, relative RLIP76 mRNA level decreased to $0.277 \pm 0.016(\mathrm{P}<0.05)$ in $\mathrm{KD}$ cells (Fig. 1F).

RLIP76 knockdown decreases the $I_{50}$ of VCR in HCT-8/V. MDR cells often have decreased intracellular drug accumulation because RLIP76 could transport VCR; thus, we verified the capacity of the RLIP76-specific shRNA lentivirus to enhance the sensitivity to VCR of HCT-8/V. The $\mathrm{IC}_{50}$ of VCR in HCT-8/V cells decreased from $164.4 \pm 1.734$ to $13.95 \pm 2.008$ $(\mu \mathrm{g} / \mathrm{ml})(\mathrm{P}<0.05)$ in KD cells (Fig. $1 \mathrm{G}$ and Table $\mathrm{I})$.

Knockdown of RLIP76 decreases the migration and invasion of HCT-8/V cells. To assess the effect of RLIP76 knockdown on HCT-8/V cell migration and invasion, we performed the in vitro migration and invasion assays of KD HCT-8/V cells and the NC. Cells that migrated across the membrane were
Table I. Effect of RLIP76 knockdown on VCR cytotoxicity in HCT-8/V cells.

\begin{tabular}{lcc}
\hline & Cell line & $\mathrm{IC}_{50}$ of VCR $(\mu \mathrm{g} / \mathrm{ml})$ \\
\hline HCT-8/V & NC & $164.4 \pm 1.734$ \\
& KD & $13.95 \pm 2.008$ \\
\hline
\end{tabular}

The $\mathrm{IC}_{50}$ of VCR was significantly reduced after RLIP76 knockdown.

quantified after incubation for $24 \mathrm{~h}$ and $48 \mathrm{~h}$ respectively. Cell migration decreased from $329.67 \pm 20.23$ to $176.33 \pm 2.52(\mathrm{P}<0.05)$ and cell invasion reduced from $294.67 \pm 30.07$ to $153 \pm 22.11$ $(\mathrm{P}<0.05)$, suggesting that RLIP76 knockdown significantly suppressed the migration and invasion of colorectal cancer cells (Fig. 2A-C). Before this, we performed another Transwell 


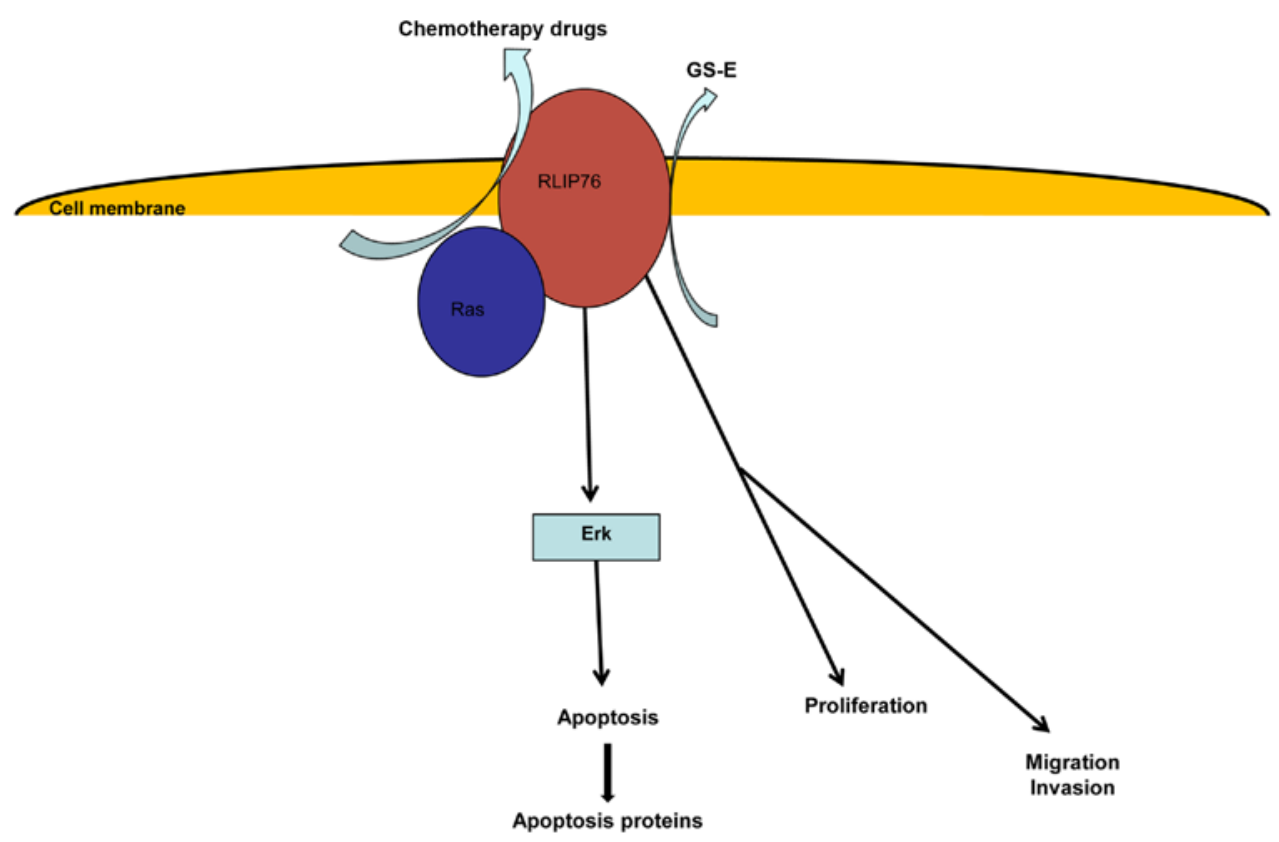

Figure 4. A model of RLIP76 functions. RLIP76 is a member of Ras family, as a result it can interact with Erk and Akt signaling pathways. RLIP76 can also transport GS-E and chemotherapy drugs, which may relate to drug resistance of cancer. RLIP76 plays an important role in cancer cells proliferation, apoptosis and metastasis.

assay compared with HCT-8 in migration and invasion. The migration and invasion level did not decrease similarly to HCT-8 even though the RLIP76 was knocked down (Fig. 2D).

RLIP76 knockdown decreases growth and increases apoptosis of HCT-8/V cells through downregulating Erk phosphorylation. We performed a CCK-8 assay to investigate the biological function of RLIP76 on cell proliferation. Knockdown of RLIP76 decreased the growth of cancer cells (Fig. 3A; $\mathrm{P}<0.05$ ). Apoptosis is a process of programmed cell death that occurs in multi-cellular organisms. In the present study, we analyzed the expression levels of Bax, caspase-3, PARP, caspase- 8 and caspase- 9 by western blot analysis. We found that the protein levels of caspase-3 decreased, whereas the Bax, cleaved-PARP, cleaved-caspase- 8 and cleaved-caspase- 9 increased in KD cells compared with the control (Fig. 3B).

The MAPK signaling pathways are well-known as important signaling pathways for cancer cell growth. To identify the potential molecular mechanisms of RLIP76 knockdown in HCT-8/V cell proliferation and apoptosis, we analyzed the expression levels of signaling proteins by western blot analysis and found that RLIP76 knockdown markedly reduced the phosphorylated-Erk from $75.8 \pm 3.02$ to $50.8 \pm 7.02 \%(\mathrm{P}<0.05)$, however, Erk protein level remained constant (Fig. 3C and D).

\section{Discussion}

Colorectal cancer is one of the most common causes of cancer- related deaths in developed countries (39). For colorectal cancer, chemotherapy is a vital prevention and treatment method. Even though significant advances have been achieved in recent years, resistance to chemotherapy is still a major problem (40). The main reasons for chemotherapy failure are insufficient intratumoral drug concentration, intrinsic overexpression of drug efflux trans- porters in tumor cells and tumor microenvironment-related factors $(9,41,42)$. The present study used wild-type, HCT-8 and MDR colorectal cancer cells, HCT-8/VCR, to investigate the molecular mechanisms and cellular behavior involved in VCR resistance. A significant finding of the present study was that RLIP76 knockdown could reduce $\mathrm{IC}_{50}$ of HCT-8/V to VCR. We developed a model to describe the functions of RLIP76, which shows that RLIP76 may play a role in chemotherapy drug transportation, cancer cell apoptosis, proliferation and metastasis (Fig. 4).

Sui et al (43) used VCR to prove that JNK or COX-2 inhibition increased intracellular VCR accumulation and the sensitivity to VCR in HCT-8/V cells. In addition, in the present study, we showed that RLIP76 is overexpressed in MDR cancer cells compared to the wild-type cancer cells on protein level and knockdown of RLIP76 significantly also reduced $\mathrm{IC}_{50}$ of HCT-8/V to VCR. The higher resistance to VCR in HCT-8/V as compared with the HCT- 8 cells is associated with a higher RLIP76-mediated efflux of VCR in HCT-8/V. Knockdown of RLIP76 with shRNA sensitizes HCT-8/V to VCR. On the contrary, MDR is a phenotype exhibited by many cancers to develop resistance to the cytotoxic effects of many structurally divergent cytotoxic agents. Accumulation defective MDR is mediated by various transporter proteins such as MRP and Pgp (44-46). However, RLIP76, a stress-responsive, stressprotective ATP-dependent transporter, also plays an important role in chemotherapy agents and glutathione conjugate (GS-E) transport. Knockout of the mouse homolog of RLIP76 leads to $80 \%$ loss of transport capacity for GS-E, and markedly increased sensitivity to stress, xenobiotics, as well as ionizing radiation (11).

Apoptosis can be initiated via one of two pathways. In the intrinsic pathway, the cell undergoes cellular stress, whereas in the extrinsic pathway, apoptosis is caused by signals from other cells. Both pathways activate initiator caspases 
to activate executioner caspases, which consequently induce cell death by indiscriminately degrading proteins. Several studies have reported that RLIP76 depletion can increase the apoptosis induced by chemotherapy drugs by suppressing cellular transport (21). In the present study, we also found that RLIP76 knockdown in HCT-8/V cells without chemotherapy significantly increased apoptosis as compared with controls. We carried out western blot analysis to detect apoptosis proteins, including caspase-3, caspase-8, caspase-9, Parp and Bax, finding that knockdown of RLIP76 decreased caspase-3, increased cleaved-caspase-8, cleaved-caspase-9, cleaved-Parp and Bax, which implies a functional interaction between RLIP76 and the caspase pathways in colorectal cancer. This observation is consistent with previous findings that RLIP76 deletion or inhibition in animal models causes rapid, complete, and sustained regression of malignancy in human xenografts (20). The apoptotic effect of RLIP76 maybe related to Ras as it is also a member of the Ras family. As Ras is a upstream protein of Erk, a relationship between RLIP76 and Erk may exist. In addition, Erk signaling represents a primary axis of a signal relay pathway that determines the basal survival and resistance to apoptotic effects. Therefore, in the present study, we investigated the interaction of Erk and RLIP76. Knockdown of RLIP76 reduced the phosphorylation level of Erk and as a result, it can enhance the effects of some chemotherapy drugs which target Erk pathways, such as sunitinib, sorafenib and temsirolimus.

Invasion and migration are features that result in poor prognosis in colorectal cancer (47). In the present study, we found that RLIP76 knockdown significantly suppressed the invasiveness and migration of KD HCT-8/V colorectal cancer cells compared with the control cells.

Our findings show that RLIP76 is overexpressed in MDR colorectal cancer cells, and RLIP76 is a very important anticancer target that functions as an anti-apoptosis protein necessary for the survival of cancer cells. It also regulates the important signaling pathways of cancer cells, such as downregulating phosphorylation level of Erk. Though further study is still needed, RLIP76 is an important target to improve drug resistance and tumor treatment.

\section{Acknowledgements}

The present study was supported in part by grants from the National Natural Science Foundation of China (81472685), the Science and Technology Development Project of Shandong Province (2013GSF11852), the Major Science and Technology Projects of Shandong Province (2015ZDXX0802A01), the Postdoctoral Innovation Project Special Foundation of Shandong Province (201302031) and the Promotive research fund for excellent young and middle-aged scientists of Shandong Province (BS2014YY037).

\section{References}

1. Cunningham D, Atkin W, Lenz HJ, Lynch HT, Minsky B, Nordlinger B and Starling N: Colorectal cancer. Lancet 375: 1030-1047, 2010

2. Verdecchia A, Francisci S, Brenner H, Gatta G, Micheli A, Mangone L and Kunkler I; EUROCARE-4 Working Group: Recent cancer survival in Europe: A 2000-02 period analysis of EUROCARE-4 data. Lancet Oncol 8: 784-796, 2007.
3. Kong Y, Bai PS, Sun H, Nan KJ, Chen NZ and Qi XG: The deoxycholic acid targets miRNA-dependent CAC1 gene expression in multidrug resistance of human colorectal cancer. Int J Biochem Cell Biol 44: 2321-2332, 2012.

4. Yasunaga M and Matsumura Y: Role of SLC6A6 in promoting the survival and multidrug resistance of colorectal cancer. Sci Rep 4: 4852, 2014.

5. Nooter K and Sonneveld P: Clinical relevance of P-glycoprotein expression in haematological malignancies. Leuk Res 18: 233-243, 1994.

6. Leith C: Multidrug resistance in leukemia. Curr Opin Hematol 5: 287-291, 1998.

7. Sharma R, Awasthi YC, Yang Y, Sharma A, Singhal SS and Awasthi S: Energy dependent transport of xenobiotics and its relevance to multidrug resistance. Curr Cancer Drug Targets 3: 89-107, 2003.

8. Takara K, Sakaeda T and Okumura K: An update on overcoming MDR1-mediated multidrug resistance in cancer chemotherapy. Curr Pharm Des 12: 273-286, 2006.

9. Jullien-Flores V, Dorseuil O, Romero F, Letourneur F, Saragosti S, Berger R, Tavitian A, Gacon G and Camonis JH: Bridging Ral GTPase to Rho pathways. RLIP76, a Ral effector with CDC42/Rac GTPase-activating protein activity. J Biol Chem 270: 22473-22477, 1995 .

10. Park SH and Weinberg RA: A putative effector of Ral has homology to Rho/Rac GTPase activating proteins. Oncogene 11: 2349-2355, 1995.

11. Awasthi S, Cheng J, Singhal SS, Saini MK, Pandya U, Pikula S, Bandorowicz-Pikula J, Singh SV, Zimniak P and Awasthi YC: Novel function of human RLIP76: ATP-dependent transport of glutathione conjugates and doxorubicin. Biochemistry 39: 9327-9334, 2000.

12. Singhal SS, Singhal J, Nair MP, Lacko AG, Awasthi YC and Awasthi S: Doxorubicin transport by RALBP1 and ABCG2 in lung and breast cancer. Int J Oncol 30: 717-725, 2007.

13. Singhal SS, Sehrawat A, Sahu M, Singhal P, Vatsyayan R, Rao Lelsani PC, Yadav S and Awasthi S: Rlip76 transports sunitinib and sorafenib and mediates drug resistance in kidney cancer. Int J Cancer 126: 1327-1338, 2010.

14. Singhal SS, Sehrawat A, Mehta A, Sahu M and Awasthi S: Functional reconstitution of RLIP76 catalyzing ATP-dependent transport of glutathione-conjugates. Int J Oncol 34: 191-199, 2009.

15. Drake KJ, Singhal J, Yadav S, Nadkar A, Pungaliya C, Singhal SS and Awasthi S: RALBP1/RLIP76 mediates multidrug resistance. Int J Oncol 30: 139-144, 2007.

16. Awasthi S, Singhal SS, Srivastava SK, Zimniak P, Bajpai KK, Saxena M, Sharma R, Ziller SA III, Frenkel EP and Singh SV: Adenosine triphosphate-dependent transport of doxorubicin, daunomycin, and vinblastine in human tissues by a mechanism distinct from the P-glycoprotein. J Clin Invest 93: 958-965, 1994.

17. Awasthi S, Singhal SS, Pandya U, Gopal S, Zimniak P, Singh SV and Awasthi YC: ATP-Dependent colchicine transport by human erythrocyte glutathione conjugate transporter. Toxicol Appl Pharmacol 155: 215-226, 1999.

18. Awasthi S, Singhal SS, Singhal J, Yang Y, Zimniak P and Awasthi YC: Role of RLIP76 in lung cancer doxorubicin resistance: III. Anti-RLIP76 antibodies trigger apoptosis in lung cancer cells and synergistically increase doxorubicin cytotoxicity. Int J Oncol 22: 721-732, 2003.

19. Yadav S, Zajac E, Singhal SS, Singhal J, Drake K, Awasthi YC and Awasthi S: POB1 over-expression inhibits RLIP76-mediated transport of glutathione-conjugates, drugs and promotes apoptosis. Biochem Biophys Res Commun 328: 1003-1009, 2005.

20. Singhal SS, Singhal J, Yadav S, Dwivedi S, Boor PJ, Awasthi YC and Awasthi S: Regression of lung and colon cancer xenografts by depleting or inhibiting RLIP76 (Ral-binding protein 1). Cancer Res 67: 4382-4389, 2007.

21. Singhal SS, Roth C, Leake K, Singhal J, Yadav S and Awasthi S: Regression of prostate cancer xenografts by RLIP76 depletion. Biochem Pharmacol 77: 1074-1083, 2009.

22. Singhal SS, Awasthi YC and Awasthi S: Regression of melanoma in a murine model by RLIP76 depletion. Cancer Res 66: 2354-2360, 2006.

23. Leake K, Singhal J, Nagaprashantha LD, Awasthi S and Singhal SS: RLIP76 regulates PI3K/Akt signaling and chemo-radiotherapy resistance in pancreatic cancer. PLoS One 7: e34582, 2012.

24. Wang Q, Qian J, Wang J, Luo C, Chen J, Hu G and Lu Y: Knockdown of RLIP76 expression by RNA interference inhibits invasion, induces cell cycle arrest, and increases chemosensitivity to the anticancer drug temozolomide in glioma cells. J Neurooncol 112: 73-82, 2013. 
25. Singhal SS, Yadav S, Vatsyayan R, Chaudhary P, Borvak J, Singhal J and Awasthi S: Increased expression of cdc2 inhibits transport function of RLIP76 and promotes apoptosis. Cancer Lett 283: 152-158, 2009.

26. Hu Y and Mivechi NF: HSF-1 interacts with Ral-binding protein 1 in a stress-responsive, multiprotein complex with HSP90 in vivo. J Biol Chem 278: 17299-17306, 2003.

27. Wang Q, Wang JY, Zhang XP, Lv ZW, Fu D, Lu YC, Hu GH, Luo C and Chen JX: RLIP76 is overexpressed in human glioblastomas and is required for proliferation, tumorigenesis and suppression of apoptosis. Carcinogenesis 34: 916-926, 2013.

28. Jullien-Flores V, Mahé Y, Mirey G, Leprince C, MeunierBisceuil B, Sorkin A and Camonis JH: RLIP76, an effector of the GTPase Ral, interacts with the AP2 complex: Involvement of the Ral pathway in receptor endocytosis. J Cell Sci 113: 2837-2844, 2000.

29. Quaroni A and Paul EC: Cytocentrin is a Ral-binding protein involved in the assembly and function of the mitotic apparatus. J Cell Sci 112: 707-718, 1999.

30. Awasthi S, Singhal SS, Sharma R, Zimniak P and Awasthi YC: Transport of glutathione conjugates and chemotherapeutic drugs by RLIP76 (RALBP1): A novel link between G-protein and tyrosine kinase signaling and drug resistance. Int J Cancer 106 635-646, 2003

31. Awasthi S, Singhal SS, Awasthi YC, Martin B, Woo JH, Cunningham CC and Frankel AE: RLIP76 and Cancer. Clin Cancer Res 14: 4372-4377, 2008.

32. Awasthi YC, Singhal SS, Gupta S, Ahmad H, Zimniak P, Radominska A, Lester R and Sharma R: Purification and characterization of an ATPase from human liver which catalyzes ATP hydrolysis in the presence of the conjugates of bilirubin bile acids and glutathione. Biochem Biophys Res Commun 175: 1090-1096, 1991.

33. Awasthi S, Singhal SS, Singhal J, Cheng J, Zimniak P and Awasthi YC: Role of RLIP76 in lung cancer doxorubicin resistance: II. Doxorubicin transport in lung cancer by RLIP76. Int J Oncol 22: 713-720, 2003.

34. Yadav S, Singhal SS, Singhal J, Wickramarachchi D, Knutson E, Albrecht TB, Awasthi YC and Awasthi S: Identification of membrane-anchoring domains of RLIP76 using deletion mutant analyses. Biochemistry 43: 16243-16253, 2004.

35. Stuckler D, Singhal J, Singhal SS, Yadav S, Awasthi YC and Awasthi S: RLIP76 transports vinorelbine and mediates drug resistance in non-small cell lung cancer. Cancer Res 65: 991-998, 2005 .

36. Singhal SS, Yadav S, Singhal J, Zajac E, Awasthi YC and Awasthi S: Depletion of RLIP76 sensitizes lung cancer cells to doxorubicin. Biochem Pharmacol 70: 481-488, 2005.
37. Awasthi S, Cheng JZ, Singhal SS, Pandya U, Sharma R, Singh SV, Zimniak P and Awasthi YC: Functional reassembly of ATP-dependent xenobiotic transport by the $\mathrm{N}$ - and C-terminal domains of RLIP76 and identification of ATP binding sequences. Biochemistry 40: 4159-4168, 2001.

38. Su JL, Lin MT, Hong CC, Chang CC, Shiah SG, Wu CW, Chen ST, Chau YP and Kuo ML: Resveratrol induces FasL-related apoptosis through Cdc42 activation of ASK1/ JNK-dependent signaling pathway in human leukemia HL-60 cells. Carcinogenesis 26: 1-10, 2005

39. Rohwer $\mathrm{N}$ and Cramer T: Hypoxia-mediated drug resistance: Novel insights on the functional interaction of HIFs and cell death pathways. Drug Resist Updat 14: 191-201, 2011.

40. Wang H, Zhao L, Zhu LT, Wang Y, Pan D, Yao J, You QD and Guo QL: Wogonin reverses hypoxia resistance of human colon cancer HCT116 cells via downregulation of HIF-1 $\alpha$ and glycolysis, by inhibiting PI3K/Akt signaling pathway. Mol Carcinog 53 (Suppl 1): E107-E118, 2014.

41. Cho K, Shin HW, Kim YI, Cho CH, Chun YS, Kim TY and Park JW: Mad1 mediates hypoxia-induced doxorubicin resistance in colon cancer cells by inhibiting mitochondrial function. Free Radic Biol Med 60: 201-210, 2013.

42. Murono K, Tsuno NH, Kawai K, Sasaki K, Hongo K, Kaneko M, Hiyoshi M, Tada N, Nirei T, Sunami E, et al: SN-38 overcomes chemoresistance of colorectal cancer cells induced by hypoxia, through HIF1alpha. Anticancer Res 32: 865-872, 2012.

43. Sui H, Zhou S, Wang Y, Liu X, Zhou L, Yin P, Fan Z and Li Q: COX-2 contributes to P-glycoprotein-mediated multidrug resistance via phosphorylation of c-Jun at Ser63/73 in colorectal cancer. Carcinogenesis 32: 667-675, 2011

44. Choudhuri S and Klaassen CD: Structure, function, expression, genomic organization, and single nucleotide polymorphisms of human ABCB1 (MDR1), ABCC (MRP), and ABCG2 (BCRP) efflux transporters. Int J Toxicol 25: 231-259, 2006

45. Higgins CF: Multiple molecular mechanisms for multidrug resistance transporters. Nature 446: 749-757, 2007.

46. Sharom FJ: ABC multidrug transporters: Structure, function and role in chemoresistance. Pharmacogenomics 9: 105-127, 2008.

47. Poon RT, Fan ST, Ng IO, Lo CM, Liu CL and Wong J: Different risk factors and prognosis for early and late intrahepatic recurrence after resection of hepatocellular carcinoma. Cancer 89: 500-507, 2000

48. Singhal SS, Singhal J, Figarola J, Horne D and Awasthi S: RLIP76 targeted therapy for kidney cancer. Pharm Res 32: 3123-3136, 2015 . 